\title{
A SINGLE CASE STUDY OF THE COMMUNICATION DEVELOPMENT OF A HIGH-RISK NEONATE, FROM BIRTH TO DISCHARGE FRON A NEONATAL INTENSIVE CARE UNIT
}

\author{
Alethea McInroy and Alta Kritzinger
}

\author{
Clinic for High Risk Babies (CHRIB) \\ Centre for Early Intervention in Communication Pathology \\ Department of Communication Pathology \\ University of Pretoria, South Africa
}

\begin{abstract}
Since preterm and low birth weight infants display a high-risk for communication disorders or delays, the Neonatal Intensive Care Unit (NICU) provides the earliest opportunity where family-centered early communication intervention (ECI) services can be initiated. Extensive knowledge about high-risk neonates exists, but there appears to be limited knowledge about the emergence of early communication skills in these neonates. The aim of the study was to provide a systematic description of the successive communication developmental steps of a high-risk neonate on a weekly basis, from birth to discharge from a NICU, in order to guide further research on a larger scale. An A-type single case study design was used to collect prospective data over 14 sessions during the participant's 51 -day stay in the NICU. Using a comprehensive data-collection protocol, rich data sets were gleaned over time. The results are described as a chronology of events contributing to the participant's risk status and influencing his early communication development. The successive emergence of the different components of language skills in the participant provided new insights into the communication development of a preterm neonate and should be further investigated. An ECI programme and guidelines for implementation in the NICU are discussed as a treatment option.
\end{abstract}

Key Words: High-risk neonates, neonatal intensive care unit, neonatal communication development, early communication intervention, parent-centered approach

\section{INTRODUCTION}

Preterm and low birth weight neonates demonstrate a high-risk for developmental disorders and more specifically communication delays and disorders (Rossetti, 2001). The Neonatal Intensive Care Unit (NICU) provides the earliest opportunity where family-centered early communication intervention (ECI) services can be initiated (Rossetti, 2001). In the South African context where preterm birth and low birth weight are the primary causes of death during the perinatal period (Pattinson, 2002), initiatives, to start ECI from birth onwards are warranted in order to minimize the effects of developmental disorders or prevent potential communication developmental delays in those infants who survive.

ECI services in the form of developmentally appropriate care have been implemented in the NICU for some years and the efficacy of parent training, staff training and interventions directed at the neonates, such as appropriate oral-motor, tactile, vestibular, kinesthetic, visual and auditory stimulation, has been established (Rossetti, 2001). In addition to numerous studies on developmental interventions in the NICU and outcomes of highrisk neonates (Als, 1997; Kritzinger, 1994; Rossetti, 2001) various theoretical frameworks are offered to describe the neurobehavioral characteristics and associated environmental factors impacting on the high-risk neonate (Als, 1999; Brazelton, 1984; Cioni, Ferrari, Einspieler, Paolicelli, Barbani \& Prechtl, 1997; Klaus \& Kennell, 2001; Samerhoff. \& Fiese, 2000; Young, 1997).

Despite in-depth descriptions of high-risk neonates, their associated medical conditions and early neurobehavioral development (Als, 1999; Als, 1997; Rossetti, 2001; Young, 1997), there appears to be limited knowledge of neonatal communication development in the NICU. References to high-risk neonatal communication behaviours are mostly based on typical development or integrated in descriptions of early mother-infant interac- tion patterns or neurobehavioural development (ASHA, 2004). It appears that no systematic chronology of the earliest communication development of high-risk infants can be found in the literature. An ordered description of the successive communication developmental steps of high-risk neonates in the NICU, may be an important source of information to identify developmentally appropriate intervention needs of the neonate and the family in the NICU. According to Rossetti (2001) regular serial assessments of high-risk infants are the only means to describe developmental patterns, to measure developmental rates and change, to identify new problems, to provide information about the effects of intervention and to guide decision making during ECI.

Informed decisions regarding developmentally appropriate care options in the NICU require a specialized knowledge base of fetal and newborn brain development, medical conditions of high-risk neonates and the subsequent neuro-developmental sequelae, different aspects of developmental and neurobehavioural patterns of high-risk neonates, the NICU environment and parenting in the NICU (Rossetti, 2001). The preterm neonate is in reality a fetus developing in an extra-uterine environment and the demands of this unnatural environment may lead to maladjusted neurobehavioural development in the NICU (Als, 1999). During the critical period of rapid brain development and the development of other organ systems the preterm infant is exposed to an environment characterized by inappropriate patterns of stimulation (Young, 1997), far removed from the dark, peaceful and protected environment of the mother's uterus (Als, 1997). ECI efforts in the NICU can therefore only attempt to compensate for the infant's untimely start of extra-uterine communication development.

Birth weight and gestational age are considered the most important indicators of an infant's risk status, and later developmental disorders and delays in the child are strongly associated with these two biological risk factors (Rossetti, 2001). Various 
complications in the perinatal period, lengthened hospitalizations and separation from the primary caregivers may contribute to delayed development, and more specifically, delayed communication development (Rossetti, 2001). Developmentally appropriate ECI goals should therefore aim to create the most ideal environment viable to support the infant's development from the earliest possible time.

Consequently, family-centered ECI services are advocated, since human relationships are essential for the survival and development of high-risk neonates (Als, 1997; Klaus \& Kennell, 2001; Rossetti, 2001). A family-centered intervention approach in the NICU is essential as the parent-infant attachment process, initiated before conception and disrupted by preterm birth, needs to be restored and continued in the first days and weeks of the neonate's life (Klaus \& Kennell, 2001). Optimum attachment is important as the caregiver and neonate's abilities to establish ideal patterns of interaction increase the potential for appropriate communication development in the neonate (Rossetti, 2001). Parents' stressful experiences of caring for the neonate in the NICU may have a negative influence on their subsequent interaction with the preterm infant (Klaus \& Kennell, 2001). Interventions aimed at supporting the family are most important since parents are the primary mediators between the neonate and the healthcare system (Young, 1997).

Due to unique circumstances every high-risk neonate and family has individual needs, which emphasizes the provision of individualized ECI programmes (Als, 1999). The basis of a developmentally appropriate intervention programme rests on the premise that the neonate communicates through behavioural changes in response to physiological changes, handling or the environment (Ziev, 1999). By rendering services to the high-risk neonate, the family and the NICU staff, long-term risks for the neonate's communication development may be decreased (Rossetti, 2001).

Due to the high prevalence of preterm and low birth weight infants in South Africa it is important that speechlanguage therapists play an active role in the NICU. Knowledge about the chronology of early communication development of infants while in the NICU, may guide developmentally appropriate ECI programmes and facilitate best practice in the NICU.

The research question for the study was stated as follows: How may serial assessment data of a high-risk neonate's communication developmental progress in the NICU be used to identify the changing needs of the neonate and parents and provide guidelines for an individualized developmentally appropriate ECI programme? Although the findings of a single case study cannot be generalized to other cases or contexts, it may be used as a basis for a larger scale study and a treatment option for ECI services in the NICU.

\section{METHOD}

\section{Aims}

The main aim of the study was to provide a systematic description of the communication development of a high-risk neonate, from birth to discharge from a NICU in order to identify changing developmental needs in the infant and parents.

Specific sub-aims were identified to track and describe the following aspects regarding the participant from birth to discharge from the NICU:

- The cascading effect of biological risk factors identified in relation to the participant
- The changing physiological states, feeding behaviour, development of neurobehavioral states and the participant's changing ability to organize himself in the NICU environment

- The participant's emerging communication development

- The process of attachment and parent-neonate interaction

- The changing needs of the participant and his parents with regards to ECI

Based on the outcomes of the sub-aims a developmentally appropriate ECI programme was applied during the neonate's stay in the NICU, and will be described together with guidelines for further intervention.

\section{Research Design}

An A-type single case study research design was selected, since it is a descriptive research design that describes one participant's behaviour as it occurs in the natural environment (McReynolds \& Kearns, 1983). A longitudinal design was used since information related to a specific situation was gathered over time (Leedy \& Ormrod, 2001), mainly by means of successive prospective observations (McReynolds \& Kearns, 1983).

\section{Participants}

\section{Participant selection criteria}

- The participant had to be a patient in a NICU for the duration of the data collection period.

- The participant had to be extremely premature, i.e. $<30$ weeks (Southgate \& Pittard, 2001) as these infants are usually cared for in a NICU for a considerable amount of time (Young, 1997) so that sufficient changes could be observed over time to allow a rich description of the case (Leedy \& Ormrod, 2001).

\section{Participant description}

The characteristics of the participant are described in Table 1.

Table 1. Characteristics of participant at birth.

\begin{tabular}{|c|c|}
\hline Characteristic & Description \\
\hline 1. Age of mother & $\begin{array}{l}33 \text { years } \\
\text { (ideal age } 18.37 \text { years, Rossetti, 2001) }\end{array}$ \\
\hline 2. Mother's educational level & Tertiary education \\
\hline $\begin{array}{l}\text { 3. Position of participant in } \\
\text { family }\end{array}$ & Second child \\
\hline 4. Mother's antenatal care & \multirow{2}{*}{$\begin{array}{l}\text { Sufficient: } 3 \text { visits before } 24 \text { weeks } \\
\text { (ideal: } 5 \text { visits before } 35 \text { weeks, Rossetti, 2001) } \\
29 \text { weeks } \\
\text { (extremely premature, Southgate \& Pittard, 2001) }\end{array}$} \\
\hline 5. Duration of pregnancy & \\
\hline 6. Reason for preterm birth & Premature rupture of membranes \\
\hline 7. Means of birth & Caesarian section \\
\hline 8. Birth-weight & $\begin{array}{l}\text { 1040g (ideal: } 3400-3800 \mathrm{~g} \text { ) } \\
\text { (Very low birth weight, Southgate \& Pittard, } 2001\end{array}$ \\
\hline $\begin{array}{l}\text { 9. Apgar score: } 1 \text { min and } 5 \\
\text { min }\end{array}$ & $\begin{array}{l}4 / 10 \text { and } 6 / 10 \text { (idéal } 7 / 10-10 / 10 \text { ) } \\
\text { (Moderate asphyxia, Rossetti, 2001) }\end{array}$ \\
\hline 10. Weight for gestational age & $\begin{array}{l}\text { Small-for-gestational age } \\
\text { (ideal } 1175 \mathrm{~g} \text { for } 29 \text { weeks, Rossetti, 2001) }\end{array}$ \\
\hline
\end{tabular}


According to Table 1 the participant, who is male, presented with various risk factors for communication disorders and sensorineural hearing loss at birth. These factors include very low birth weight $(<1500 \mathrm{~g})$, extremely preterm birth $(<30$ weeks gestation), small-for-gestational age and moderate birth asphyxia (Joint Committee on Infant Hearing, 1994; Rossetti, 2001; Southgate \& Pittard, 2001). Protective factors for the participant's development were the mother's age, educational level, sufficient antenatal care and the fact that the participant was the parents' second child. The participant's parents were Setswana speaking, lived in a rural area $50 \mathrm{~km}$ from the NICU and the mother was a teacher and the father a policeman. The risks appeared to be mainly biological conditions with the participant's environment providing various protective factors that would support further development.

\section{Materials}

A comprehensive data-collection protocol, comprising of ten different developmental areas and related aspects, was compiled. Firstly, the Risk Assessment (Kritzinger, 1994) was used to compile a risk profile and secondly, the Genetic Screening Checklist (Louw \& Kritzinger, 1998) was used to describe any possible physical anomalies. Thirdly, the Developmental Assessment (Willemse, 2003) was used to describe neonatal state transitions over time as well as the increased ability of the participant to organize himself. The Neonatal Communication Assessment Instrument (Kritzinger \& Louw, 2003) was used to describe the following four areas of development, i.e. the participant's earliest stages of prelinguistic communication development (precursors of language use, content and form) and precursors of cognition. Components of the Oral-Motor/Feeding Rating Scale (Jelm, 1990) and the Feeding Evaluation Form for At-Risk Infants (FEFARI, Uys, 2000) were used to assess feeding behaviour from birth to discharge from the NICU. The ninth component of the data collection protocol was a description of the physical environment of the NICU. A component of the Neonatal Communication Assessment Guide (Swanepoel, 2000) was used for this purpose. Lastly; the Observation of Communication Interaction (Klein \& Briggs, 1987) was used to describe the development of communication interaction between the parents and the participant over time.

\section{PROCEDURES}

\section{Pilot study}

A pilot study was carried out to determine the effectiveness of the data-collection protocol in the NICU. Permission was obtained from the hospital authority to conduct the research. The selection criteria stipulated for the main study were used to select a single participant for the pilot study. Informed consent was obtained from the participant's parents. The complete datacollection protocol was administered when the participant was thirteen days old. The age of the participant ensured that a range of neonatal behaviours could already be observed.

The results of the pilot study were used to adjust the protocol as follows, thereby contributing to its validity and reliability. (Leedy \& Ormrod, 2001).

- A description of the routine activities that nurses performed on the participant while data collection took place, was added to the protocol. Routine care may elicit certain physiological and behavioural responses in a neonate (Young,
1997) and must therefore be considered when interpreting neonatal behaviour.

- The pilot study enabled the researcher to plan the duration of the data collection sessions and interview with the parents.

- Recurrent items in the data collection protocol were removed.

- The pilot study gave the researcher the opportunity to meet the NICU staff and become familiar with the routines and cultural pattems in the NICU (Rossetti, 2001; Ziev, 1999).

\section{Reliability and validity of the study}

\section{Reliability}

The following measures were taken to increase the reliability of the data-collection protocol:

- The data-collection protocol was subdivided into ten different sections so that every section could be observed and recorded separately. Reliability could be increased since the researcher could focus on a single component of the data collection protocol at a time.

- A pilot study was carried out in order to refine the data collection protocol.

- The participant for the main study was observed 14 times at regular intervals of three to four days apart and an hour was set aside for data collection.

- Only the head nurse of the NICU and another senior nurse were aware of the research project. The staff were therefore not biased to adjust their routine care-giving activities and pay special attention to the participant.

\section{Validity}

The following types of validity were considered in the study:

Face validity refers to the appearance of the instrument and the question is whether the appearance confirms those variables which the data collection protocol must measure (De Vos, 1998). To improve the face validity the protocol was given to a nurse with extensive experience in developmental care in the NICU for comments and her recommendations were integrated into the protocol.

Internal validity refers to the researcher's ability to make accurate deductions concerning the data collected (Leedy \& Ormrod, 2001). The researcher ensured that deductions were based only on the data that was collected (Leedy \& Ormrod, 2001 ) and that it could be compared to other research findings.

\section{Ethics}

The research proposal of the study was approved by the Research Ethics Committee of the Department of Communication Pathology, University of Pretoria. Ethical issues taken into consideration during this study can be categorized as follows (De Vos, 1998; Leedy \& Ormrod, 2001):

\section{Non-maleficence:}

Care-giving routines were not interfered with, the participant was not handled and hospital regulations concerning control of infection were observed. Recommendations were made to test the participant's hearing and to obtain ECI services for the family. Informed consent for both the pilot and the research study were obtained from the participants' parents.

The right to privacy: The participants' parents maintained their rights about any personal information to be included in the study. 


\section{Making results known:}

The researcher made the results known in an accurate and honest manner by means of the following:

- Naming shortcomings and further research possibilities.

- Results were made known in an accurate, objective and distinct way.

- All resources were acknowledged.

- Upon request the results were made known to the hospital and the participant's parents in the main study.

\section{Confidentiality:}

No identifying information about the participants or parents was included in the final report.

\section{Data collection and data analysis}

Data was recorded twice per week over 14 sessions, from the participant's birth to discharge from the NICU, using the complete data-collection protocol for every session.

Data was obtained from the participant's medical file, an interview with the parents and direct observation of the participant and parents.

The mother-infant interaction was observed eight times, while two opportunities arose to observe father-infant interaction.

The observation of parent-infant interaction depended on the availability of the participant's parents to visit him at times that were also convenient for the researcher.

The successive data sets of the single participant were organized into different categories based on themes, concepts and corresponding characteristics (De Vos, 1998).

Objective measurements were obtained by calculating frequencies and averages of the participant's functioning on the different components of the data collection protocol, which were represented in tables, graphs and figures.

\section{RESULTS AND DISCUSSION}

The cascading effect of events leading to the biological risk status of the participant A summary of successive events starting during the mother's pregnancy, leading to the participant's preterm birth and subsequent stay of 51 days in the NICU is presented in Figure 1.

According to Figure 1, and also indicated earlier in Table 1, a series of biological events prenatally, lead to the participant's highrisk status. It is well known that extremely preterm birth and very low birth weight in a neonate may lead to the conditions which also occurred in the participant. The participant experienced respiratory distress syndrome, sepsis and anaemia, and subsequent medical interventions and medications, some of which could be damaging to developing organs such as the cochlea (Joint Committee on Infant Hearing, 1994).

It appears that a risk assessment represented as a chronology of events in Figure 1 may serve as a useful technique for speech-language therapists working in NICU's to keep track of conditions that may have long-term developmental implications for their clients. Instead of viewing the different risk factors as separate events in the mother and the infant, a chronology serves to provide a multidimensional perspective on the inter-relatedness of the different risk conditions. According to Shprintzen (1997) the trajectory of early biological events over time, expressed as the cascading effect of gene expression and incidents early in life, may be used to gain insight into the causes of genetic syndromes and developmental disabilities in children. The concept of continuum of risk, described by Rossetti (2001) expresses the same principle, but extends into the postnatal period and further in life. This concept may assist clinicians in ECI to consider retrospective factors relating to the currently identifiable causes of, and contributing factors to the communication disorders or delays in their clients.

The different events determining the participant's risk status in Figure 1 were used as a framework to analyze and interpret data about the participant's physiological states, feeding behaviour, and developmental stages in the NICU.

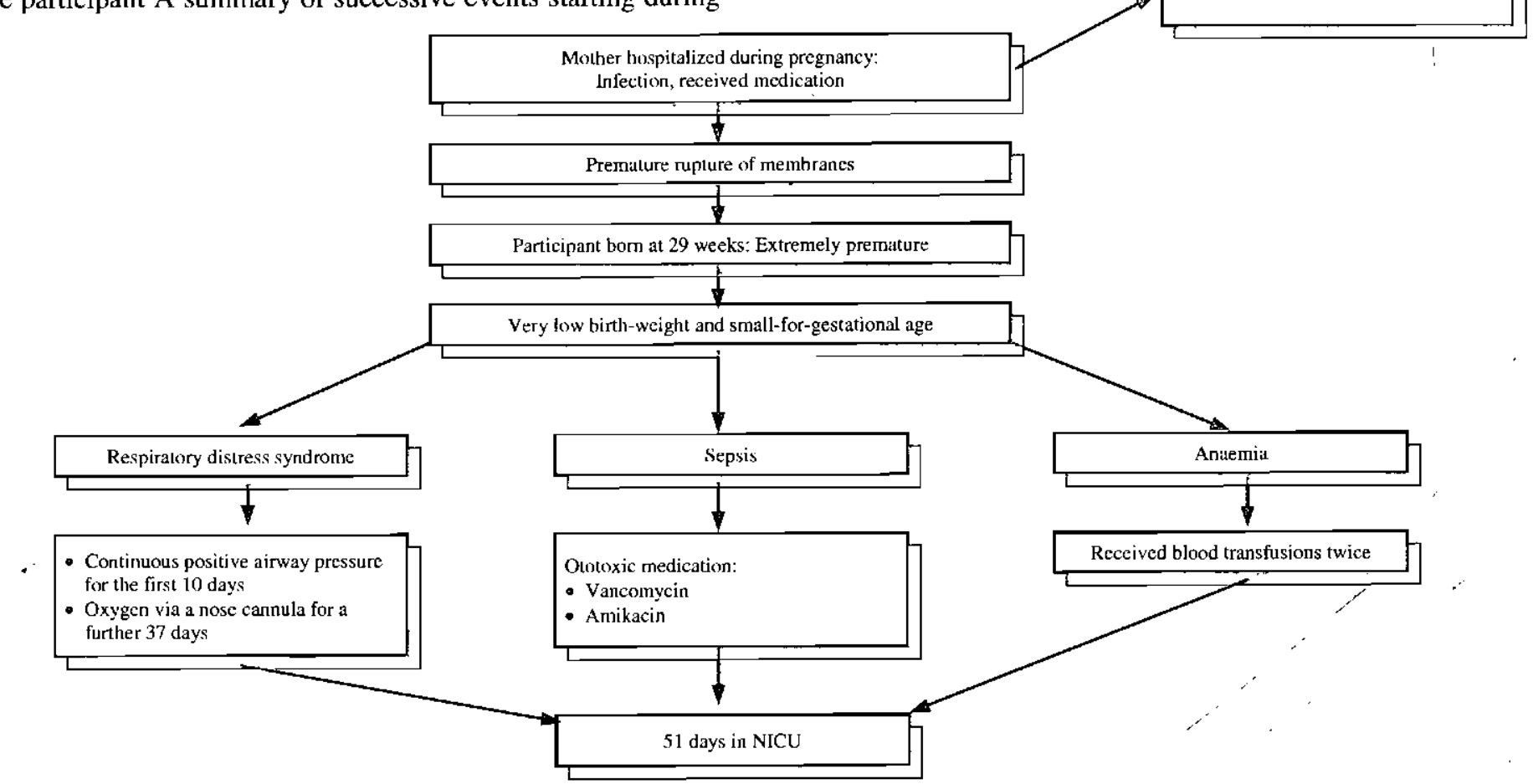

Figure 1. The cascading effect of the biological risk factors of the participant resulting in the 51 day stay in the NICU. 
The changing physiological states, feeding behaviour and developmental stages of the participant while nursed in the NICU environment
A detailed analysis of the chronology of the different developmental aspects underlying the participant's communication development is provided in Table 2 .

Table 2. The physiological states, feeding behaviour and states of the participant in the NICU environment.

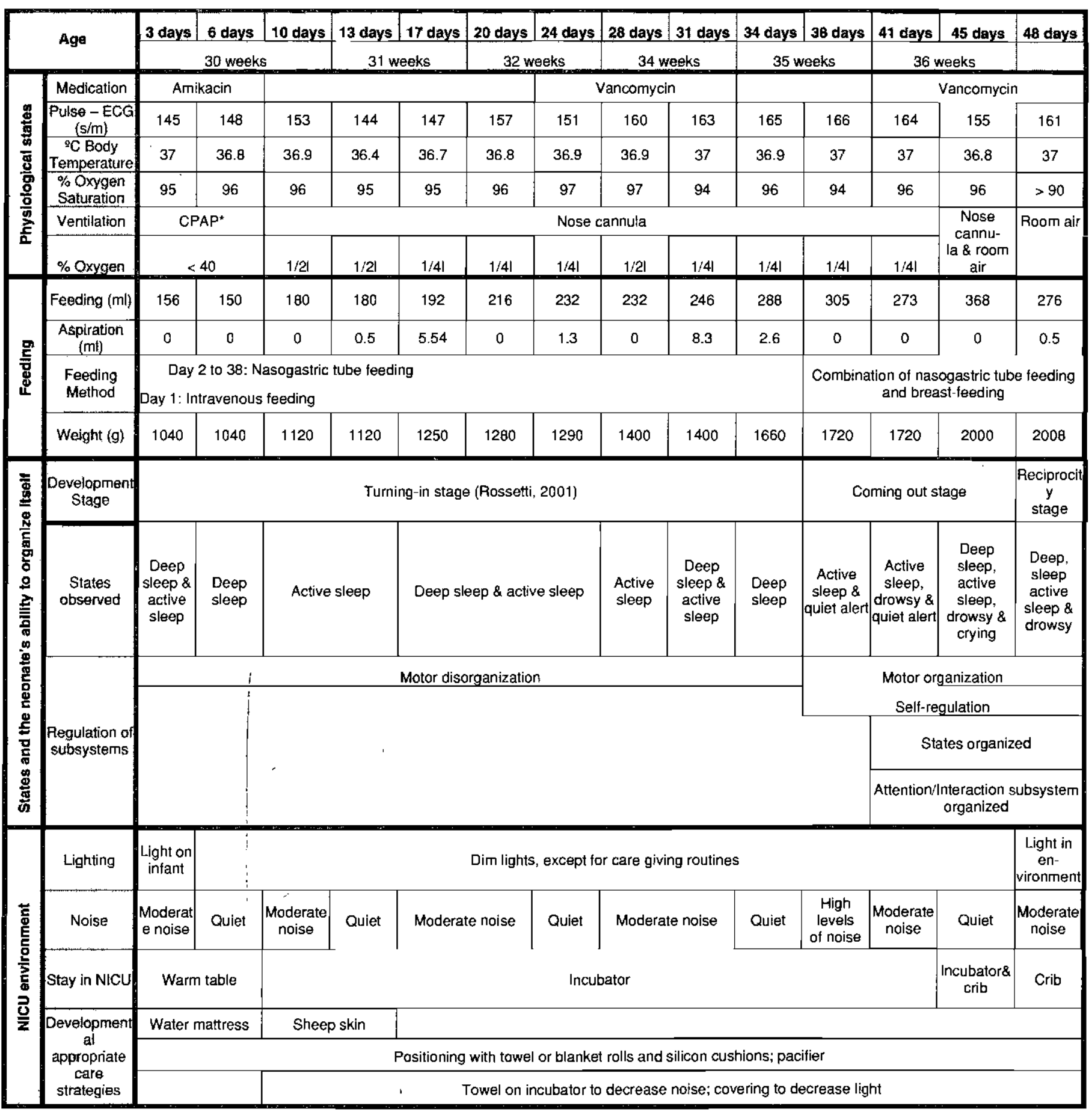

Note: *Continuous Positive Airway Pressure 
The results suggest that the participant, even though extremely premature, was kept physiologically stable from birth to discharge by the life support interventions that have increased the survival rates of high-risk infants in the NICU in recent years (Als, 1997). As indicated in Table 2 the participant's body temperature was stable and oxygen saturation levels were above the recommended 90\% levels (Zaichkin, 1996). The participant's physiological stability resulted in maturation that could be observed in the successful transition from nasogastric tube feeding to breast-feeding during the last 13 days of his stay in the NICU. Fairly consistent weight gain in relation to the amount of fluid taken per day was also observed in the participant.

Upon close inspection of Table 2 it can be seen that a pacifier was provided to facilitate nutritive sucking in the participant, but since no further feeding intervention was implemented by the nursing staff, an opportunity for feeding therapy guidelines was missed. Since the participant was physiologically stable, guidelines for optimal positioning and oral stimulation and the early introduction of Kangaroo Mother Care may have facilitated successful breast-feeding at an earlier stage in the participant (Van Rooyen, Pullen, Pattinson \& Delport, 2002).

As depicted in Table 2 the participant went through the neuro-developmental stages described by Gorski, Davidson and Brazelton in 1979 (in Rossetti, 2001). The turning-in stage usually occurs in infants less than 32 weeks gestation age, and the participant's state of awareness typically fluctuated between deep and active sleep and he was easily startled by environmental stimuli such as noises, which could be observed as motor disorganization (Rossetti, 2001). Characteristics of motor disorganization displayed by the participant included jerky movements, large movements away from the body, hyperextension, repetitive uncoordinated movements and poor sucking (Gardner \& Lubchenco, 1998).

The participant's physiological state was mechanically maintained and he displayed no self-regulatory behaviour during the turning-in period of data collection. No regulation of the other subsystems, such as the attention/interaction, motor and state regulatory subsystems occurred (Gardner \& Lubchenco, 1998; Willemse, 2003).

The participant reached the coming-out stage at 38 weeks gestation age, a stage typically reached after 35 weeks
(Rossetti, 2001). Although delayed, the coming-out stage was characterized by a greater variety of awareness states in the participant, thereby demonstrating his increased ability to regulate and organize himself in the extra-uterine environment. During the coming-out and reciprocity stages the participant experienced five of the six states of awareness, from deep and active sleep, to drowsy, quiet alert and crying (Rossetti, 2001). No active alert state was observed during any of the data collection sessions.

The NICU environment appeared to provide some support to the participant's development since lights were mostly dimmed, coverings were used to decrease light, and developmentally supportive equipment such as a water mattress, a sheep skin, blanket rolls, silicon cushions and a pacifier was provided (Zaichkin, 1996). The participant's mother was encouraged to practice Kangaroo Mother Care by the nursing staff when the participant was no longer receiving continuous positive airway pressure. It is not known to what extent the mother received training to sustain this excellent technique, now considered as best practice for high-risks neonates (Power, 2002). The moderate to high noise levels occurring regularly during datacollection times (Swanepoel, 2000) appear to be typical of descriptions of NICU environments in the literature (Gardner \& Lubchenco, 1998) and are considered as a major source of physiological stress for preterm infants (Rossetti, 2001).

Knowledge regarding NICU environmental stressors, the medical and developmentally appropriate interventions implemented in the NICU is essential to guide the speech-language therapist in designing comprehensive ECI programmes (Rossetti, 2001). ECI programmes must include strategies to protect highrisk neonates from noise bombardment, facilitate their communication development, involve their parents from the beginning and compensate for the separation from their primary caregivers.

\section{The participant's communication development in the NICU}

The tracking of the participant's communication development, described in terms of precursors of language content, form, use and cognition, was carried out as serial assessments, using the Neonatal Communication Assessment Instrument (Kritzinger \& Louw, 2003). The results are presented in Figure 2.

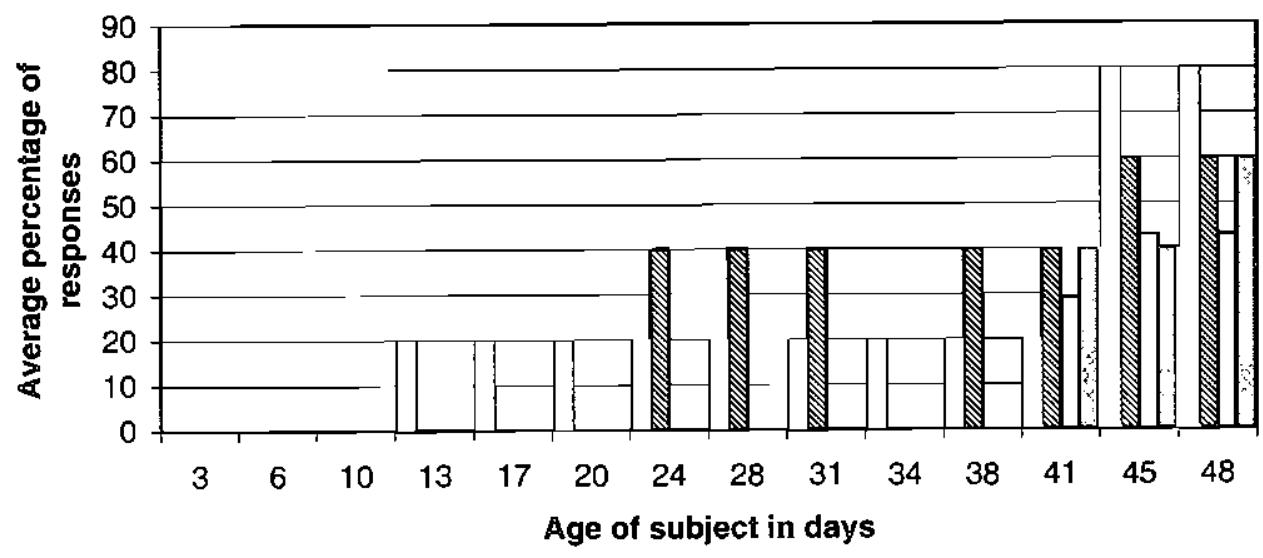

$\square$ Precursors to language content $\quad$ Precursors to language form 口Precursors to language use Derecursors to cognition

Figure 2. The communication development of the participant during his stay in the NICU. 
According to Figure 2 the participant's communication development gradually unfolded as the different components of language skills emerged. Precursors of language content appeared first on Day 12, when the participant started to respond reflexively to sound during the turning-in neurodevelopmental stage (See Table 2). During the participant's turning-in stage on Day 24 , the second component of language to emerge were the precursors of language form, characterized by the emergence of differentiated cries, crying in response to another infant's cry, and discomfort sounds. After Day 38 the participant progressed to the coming-out stage, displaying increased levels of motor organization, self-regulation and feeding skills (See Table 2), concurrently with the emergence of precursors of language use and cognition. At this stage the participant made fleeting eye contact with caregivers, accepted cuddling, displayed adapting behaviours after repetitive stimuli, and demonstrated selfcomforting responses. Upon discharge from the NICU after 51 days the participant did not yet exhibit all the communication interaction skills typical of a full-term newborn infant (Kritzinger, 1994), thereby displaying a risk for a communication disorder or delay.

The successive emergence of the different components of language skills in the participant provided new insights into the communication development of a preterm neonate and should be further investigated in a representative sample so that reliable inferences can be made. The emergence of hearing responses only on Day 12, followed by differentiated vocal expressions several days later, and interactive communication skills at a later stage in the participant, appear to correspond with certain fetal developmental patterns as described by Lecanuet, Granier-Derferre and Busnel (1995) and Gottlieb (1971, in ASHA, 2004). Hearing responses and prenatal auditory learning are present in fetuses during the last trimester of pregnancy (Lecanuet, et al., 1995), the first vocal expression occurs only at birth with the birth cry, and interactive communication behaviours are characteristic of newborn communication capabilities. Gottlieb (1971, in ASHA, 2004) describes the successive development of sensory systems as a process where the tactile system develops first during the fetal period, followed by the vestibular, auditory and visual systems. The implication is that the auditory system functions before the visual system, which indicates the same sequence of sensory functioning and language development observed in the participant of the present study.

The results of the successive development of communication skills in the participant provide guidelines for the gradual introduction of sensory experiences in the individualized ECI programme for the participant. Avoiding noise exposure and replacing inappropriate auditory stimulation with gradual exposure to human voices, preferably the voices of the participant's primary caregivers, appear to be developmentally appropriate. The interactive component of language development, $i$. e. language use, should only be introduced when the infant's physiological subsystems display readiness for reciprocity. Self-quieting behaviours and the ability to respond to caregivers are indicators that the infant is ready for reciprocal interaction (Rossetti, 2001). According to Rossetti (2001) sensory over-stimulation may prolong the duration of an infant's time in the turning-in stage. ECI strategies and techniques to promote high-risk neonates' progress through the different stages and the key role that their parents can play in early communication development, should therefore be investigated.

\section{Progress of parent-infant interaction during the participant's} stay in the NHCU

The Observation of Communication Interaction (Klein \& Briggs, 1987) was used to measure the quality of the interactions between the parents and the participant. Since the parents lived $50 \mathrm{~km}$ from the hospital, they could not visit the participant regularly. The results are depicted in Figure 3.

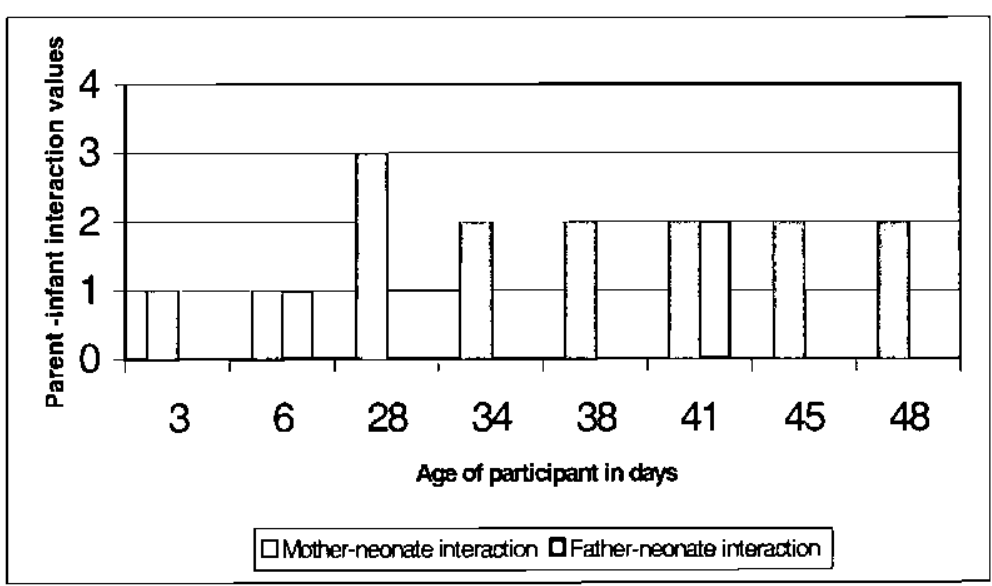

Figure 3. Parent-infant interaction during the participant's stay in the NICU. Note: Parent-infant interaction values: $1=$ never; $2=$ sometimes; $3=$ often; $4=$ optimally

As indicated in Figure 3 the participant's parents both displayed limited interaction with their infant during the first observation sessions, despite the nurses' encouragement to talk to the infant and to provide appropriate tactile stimulation. Both parents read the information file provided by the NICU staff to encourage parental participation in the care-giving routines of the infants, and read the ECI plans provided weekly by the researcher. When the participant was 28 days old the frequency of the mother's interaction with the participant during data collection could be described as "often" (See Figure 3, key to parent-infant interaction measurement), even though he was still in the turning-in stage and not responding actively to her interest shown in him. No specific reason for the mother's increased interaction on that day with the participant can be offered, except that both the NICU staff and the researcher continuously encouraged her involvement in the participant.

According to the results there was an increase in parent-infant interaction over time, but could only be described as "sometimes" and did not change when the participant emerged from his turning-in stage. Although the neonate's level of arousal increased according to the corresponding developmental stage, the type and amount of input given to the infant by the parents was not increased. Consequently, appropriate interaction between the participant and his parents was not facilitated.

The quality of the parent-infant interaction sessions must be viewed against the extremely stressful experiences of parents while their infants are in the NICU (Güldenpfennig, 2000). The normal attachment process that already starts during pregnancy and must be continued after birth so that interaction can develop, is interrupted by the participant's preterm birth and need for immediate medical care (Klaus \& Kennell, 2001). Apart from the delayed attachment process, parents must adapt to the strange environment of the NICU, lack of privacy and often experience feelings of anxiety, fear, concern, guilt, aggression, denial, helplessness and depression (Güldenpfennig, 2000). These feelings contribute to the parents' uncertainty about their role in the NICU. Feelings of helplessness may 
disempower parents which may limit their involvement with their infant (Güldenpfennig, 2000).

It is clear that insight into parental emotions and experiences are essential so that valid interpretations of the participant's parents' lack of interaction with him can be made. Parental perceptions of the role of professionals and their respect for those professionals are culturally based and may influence their involvement in ECI (Louw \& Avenant, 2002). It could also be that the parents were not treated as autonomous members of the decision making team in the NICU, which could influence the power balance between professionals and parents. The participant's parents were experienced caregivers, since he was their second child, but they required a sensitive family-centered approach to support them through the process of having a medically fragile infant in the NICU and they needed training to appropriately stimulate the development of the infant.

The changing needs of the high-risk neonate and parents form birth to discharge form the NICU

The results of the study were integrated in an overview of the changing needs and opportunities for developmentally appropriate care strategies that will also enhance the early communication development of the participant. The participant advanced through the different neurodevelopmental stages of turning-in, coming out and reciprocity as described in the literature (Rossetti, 2001). Each of these stages requires distinct changes that should be reflected in the individualized ECI programme for the participant and his parents. The parents of the participant displayed the following needs:
- Emotional support, especially when the participant was in the turning-in stage of development. The parents required counseling from professionals in the NICU, information on their infant's conditions and capabilities, and training to improve their interaction skills with the participant and to gain confidence.

- Encouragement to visit the participant more often, since they lived far from the hospital.

- Development of skills to interact with the participant according to his developmental stages. Since the parents displayed limited interaction with their infant despite continued guidance, demonstration and direct modeling of appropriate interaction with the participant by the nurses and the speechlanguage therapist is recommended. Factors hampering their full participation should be considered.

- Use of Kangaroo Mother Care and breast-feeding times for communication interaction between mother and infant. The mother should gain insight into the fine balance between over-stimulation and appropriate stimulation techniques.

- Discharge planning in order to facilitate the take over of complete care of the participant in a developmentally appropriate way.

ECI service delivery in the NICU is guided by the development of the high-risk neonate, which in turn impacts on the parents, staff and regulation of the environment. Based on the results of the study an example of a developmentally appropriate communication intervention programme in the NICU, that may be used as a treatment option, is provided in Appendix A. The different developmental areas and stages to be considered in the compilation of such a programme are summarized in Figure 4.

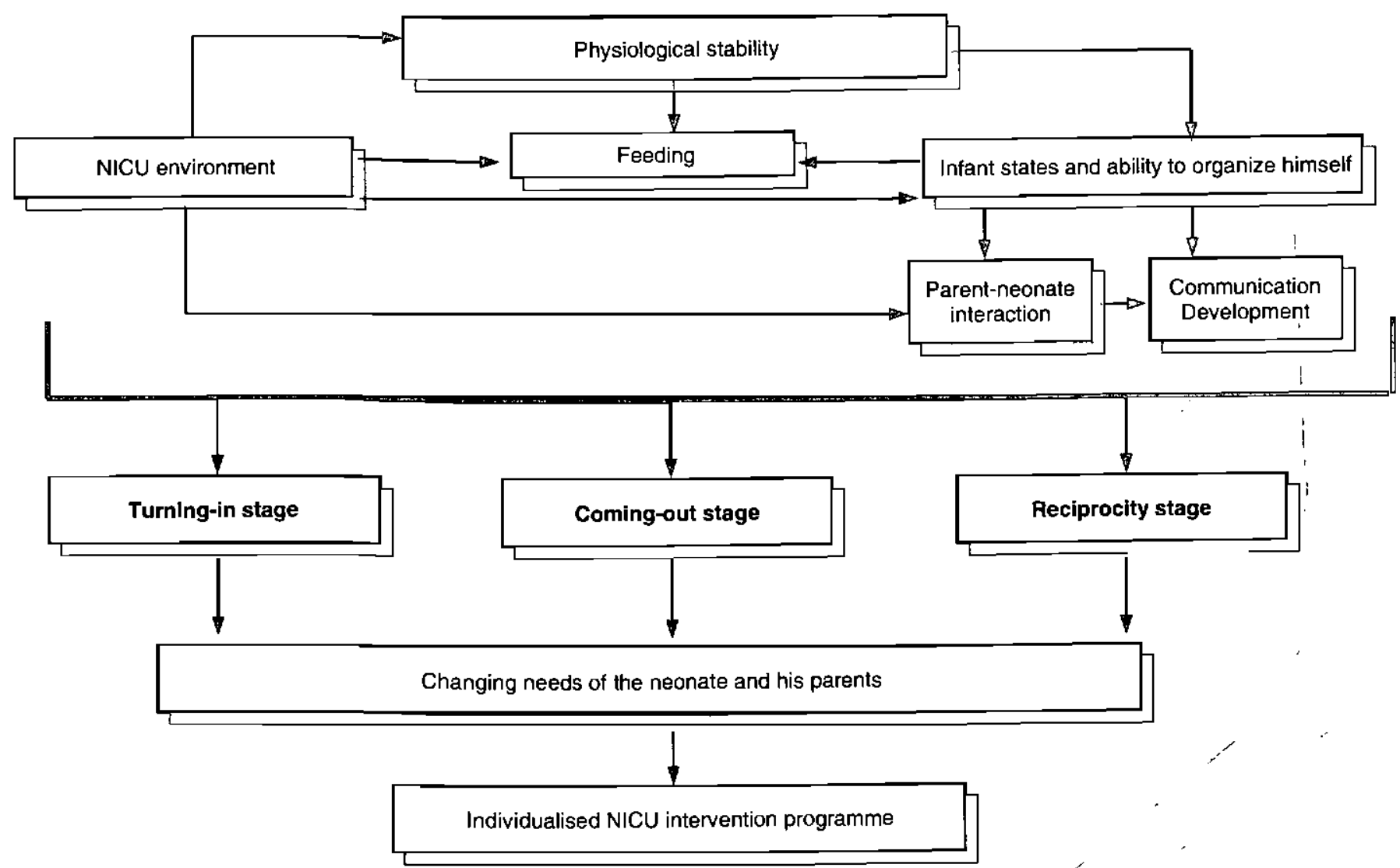

Figure 4. The interrelationship between the developmental areas and stages of the participant which may guide the NICU intervention programme 
The ECI programme presented in Appendix A is based on the principles of individualized care and a family-centered approach (Als, 1997; Rossetti, 2001), with the aim to ensure the best possible outcomes for the infant and family. The guidelines include a wide variety of topics to provide parents with information to become more familiar with the highly technical environment of the NICU, their infant's medical conditions and behaviour patterns. The information is mainly provided as handouts, but may be discussed with the parents as well. Finally, the ECI programme is holistic and interventions are directed at the infant, parents and the staff of the NICU. As indicated by Gottlieb (1971, in ASHA, 2004) and Lecanuet, et al. (1995), and emphasized by the results of the study, direct intervention with the high-risk neonate involves the successive introduction of sensory stimuli as the infant moves through the turning-in, coming out and reciprocity stages of development.

\section{CONCIUSION}

The description of the early successive communication development of a single high-risk neonate provided rich and new information that should be further investigated. Due to the limitations of a single case study the results cannot be generalized, but may serve as an example of a case that may be encountered in clinical practice. The results describing the effect of the preterm birth and NICU experience on the participant's parents should alert clinicians to the sensitive approach and skills required when working with parents, without whose full participation ECI efforts cannot be effective (Rossetti, 2001).

The developmentally appropriate communication intervention programme may be adapted to suit the unique needs of high-risk neonates and their families in private and public health settings in South Africa. The results also serve to alert speechlanguage therapists and audiologists of the set of events that contributed to the communication development of an infant or child and family who spent time in an NICU and who now requires their services. The urgency for knowledge about highrisk neonates and their families is now more important than ever in South Africa since poverty and the increased incidence of low birth weight and preterm infants among pregnant women witli HIV/AIDS (Van Rooyen, et al., 2002) requires the attention of all involved in early intervention efforts.

\section{REFERENCES}

Als, H. (1997). Earliest intervention for preterm infants in the newborn intensive care unit. In M. J. Guralnick, (Ed.), The Effectiveness of Early Intervention ( $2^{\text {nd }}$ ed., pp. 4776). Baltimore: Paul H. Brooks Publishing Co.

Als, H. (1999). Reading the premature infant. In E. Goldson (Ed.), Developmental Interventions in the Neonatal Intensive Care Nursery (pp. 18-85). New York: Oxford University Press.

ASHA, (2004). Roles of speech-language pathologists in the neonatal intensive care unit: Technical report. Retrieved September 1, 2004, from http://www.asha.org/members/ deskrefjournals/deskref/default

Brazelton, T.B. (1984). Neonatal Behavioural Assessment Scale ( $2^{\text {nd }}$ ed.). London: Spastics International Medical Publications.
Cioni, G., Ferrari, F., Einspieler, C., Paolicelli, P.B., Barbani, M.T., \& Prechtl, H.F.R. (1997). Comparison between observation of spontaneous movements and neurologic examination in preterm infants. The Journal of Pediatrics, 130(5), 704-711.

De Vos, A.S. (1998). Research at grass roots. A primer for the caring professions (pp. 76-94). Pretoria: J.L. van Schaik Academic.

Gardner, S.L., \& Lubchenco, L.O. (1998). The neonate and the environment: Impact on development. In G.B. Merenstein, \& S.L. Gardner (Eds.), Handbook of Neonatal Intensive Care. ( $4^{\text {th }}$ ed., pp. 197-242). St Louis: Mosby, Inc.

Güldenpfennig, D. (2000). Early intervention for parents of low birth weight premature babies: Development of a programme from an educational psychological perspective. Unpublished doctoral dissertation, University of Pretoria, Gauteng, South Africa.

Joint Committee on Infant Hearing. (1994). 1994 Position statement. Audiology Today, 6(6), 6-9.

Jelm, J.M. (1990). Oral-motor/Feeding rating scale. Tucson: Therapy Skill Builders.

Klaus, M.H., \& Kennell, J.H. (2001). Care of parents. In M.H. Klaus, \& A.A. Fanaroff (Eds.), Care of the High-Risk Neonate ( $5^{\text {th }}$ ed., pp. 195-222). Philadelphia: W. B. Saunders Company.

Klein, M.D., \& Briggs, M.H. (1987). Observation of communicative interaction. Journal of Childhood Communication Disorders, 10, 95-106.

Kritzinger, A. M. (1994). Vroeë kommunikasieontwikkeling van biologiese risikobabas. Unpublished masters thesis, University of Pretoria, Gauteng, South Africa.

Kritzinger, A. (2003). Risk assessment. Department of Communication Pathology, University of Pretoria, Gauteng, South Africa.

Kritzinger, A., \& Louw, B. (2003). Clinical training of undergraduate communication pathology students in neonatal assessment and neonate-caregiver interaction in South Africa. The South African Journal of Communication Disorders, 5, 5-14.

Lecanuet, J., Granier-Deferre, C., \& Busnel, M. (1995). Human fetal auditory perception. In J. Lecanuet, W.P. Fifer, N.A. Krasnegor, \& W.P. Smotherman (Eds.), Fetal development. A psychobiological perspective (pp. 239-262). New Jersey: Lawrence Erlbaum Associates, Inc.

Leedy, P.D., \& Ormrod, J.E. (2001). Practical Research. Planning and Design. New Jersey: Prentice-Hall, Inc.

Louw, B., \& Avenant, C. (2002). Culture as context for intervention: Developing a culturally congruent early intervention program. International Pediatrics, $17(3)$, $145-$ 150.

I ouw, B., \& Kritzinger, A. (1998), Genetically based communication disorders: Technological advances relevant to the speech-language therapist and audiologist [Monograph]. Clinica: Applications in Clinical Practice of Communication Pathology, 3,11-26.

McReynolds, I.V., \& Kearns, K.P. (1983). Single-subject experimental designs in communicative disorders. Baltimore: University Park Press. 
Pattinson, R.C. (2003). Perinatal care surveys 2000-2002: An overview of the challenges. In Saving babies 2002. Third perinatal care survey of South Africa (pp. 1-4). Pretoria: The MRC Unit for Maternal and Infant Health Care Strategies.

Power, D. (2002, December). Opening address. Fourth International Workshop on Kangaroo Mother Care, Cape Town, South Africa.

Rossetti, L.M. (2001). Communication intervention. Birth to three. Canada: Singular Thomson Learning.

Samerhoff, A.J., \& Fiese, B.H. (2000). Transactional regulation: The developmental ecology of early intervention. In J.P. Shonkoff, \& S.J. Meisels, (Eds.), Handbook of early childhood intervention ( $2^{\text {nd }}$ ed., pp. 135-159). Cambridge: Cambridge University Press.

Shprintzen, R.J. (1997). Genetics, syndromes and communication disorders. San Diego: Singular Publishing Group, Inc.

Southgate, W.M., \& Pittard, W.B. (2001). Classification and physical examination of the newborn infant. In Klaus, M.H., \& Fanaroff, A.A. (Eds.), Care of the high-risk neonate. $\left(5^{\text {th }}\right.$ ed., pp.100-129). Philadelphia: W. B. Saunders Company.
Swanepoel, D. (2000). Compilation and formative evaluation of a preliminary transdisciplinary neonatal communication assessment tool. Unpublished bachelors thesis, University of Pretoria, Gauteng, South Africa.

Uys, K.J. (2000). Oral feeding skills of premature infants. Unpublished masters thesis, University of Pretoria, Gauteng, South Africa.

Van Rooyen, E., Pullen, A.E., Pattinson, $\mathbb{R} . \mathbb{C}$., \& Delport, S. D. (2002, April). The value of the kangaroo mother care unit at Kalafong Hospital. Geneeskunde. The Medicine Journal, 6-10.

Willemse, S. (2003). Developmental care plan. Medforum Hospital, Pretoria.

Young, J. (1997). Developmental care of the premature baby. London: Baillière Tindall.

Zaichkin, J. (1996). Newborn intensive care. What every parent needs to know. Santa Rosa: NICU Ink Book Publishers.

Ziev, M.S.R. (1999, May/ June). Earliest intervention: Speechlanguage pathology services in the neonatal intensive care unit. $A S H A$, pp.32-36.

\section{APPENDIX A}

\section{Developmentally appropriate communication intervention programme in the NICU}

Compiled by Alethea Mclnroy and Alta Kritzinger, 2003

\section{Principles of the programme (Willemse, 2003)}

- Individualized care of the neonate in order to improve the developmental outcomes, to support growth and development and to facilitate the stabilization of physiological functioning and behaviour

- Family-centered care that views the neonate within the family context and that involves the parents in the care of the neonate from birth to discharge from the NICU

\section{Aims of the programme (Willemse, 2003)}

- To protect and promote the developmental progress of each neonate

- To avoid harmful environmental stimulation

- To ensure the best possible outcomes for the neonate and the family

These aims can be accomplished by supporting the neonate, the family and the NICU staff.

\section{Guidelines for implementing the Communication Intervention Programme}

Guidelines were explained in a file provided to the parents. The participant's parents had access to the following information in the file that was kept at the participant's incubator:

- Individualized care plan: These care plans were compiled weekly and provided information on (Rossetti, 2001; Willemse, 2003):

- How the participant demonstrates that he is content

- Which activities and handling patterns increase the participant's stress levels and should be avoided

- Which observable behaviours demonstrate signs of stress in the participant

- Which techniques may be used to comfort the participant and facilitate his growth and development

- Suggested developmentally appropriate activities to include in the participant's schedule

- A pamphlet on the hearing abilities of high-risk neonates, risks for hearing impairment in the NICU and information on hearing testing after discharge

- Guidelines on handling the high-risk neonate for the primary caregivers and family

Phases of adaptation of the family of the high-risk neonate

Information brochure: Who is the high-risk infant?

Information about the physical NICU environment

The nature of the NICU as a social environment: Handout about Kangaroo Mother Care and a handout about the Stimulation of Early Speech, Language and Listening Skills (Handouts may be obtained from the second author)

Guidelines on "swaddling bathing"

Discharge criteria

Guidelines for caring of the high-risk neonate at home 


\section{Guidelines for ECI in the NICU}

Based on the results of the study and Rossetti (2001) and Willemse (2003) the following guidelines for ECI in the NICU are proposed:

\section{Intervention directed at the neonate}

- Appropriate positioning and handling

- Calming techniques

- Regulation of developmental subsystems

- Socio-communicative interaction

- Appropriate and graded tactile, auditory and visual stimulation: Provide auditory stimulation only when the infant is in the turning-in and coming out stages. The visual component of communication interaction may be added when the infant enters the reciprocity stage of development.

\section{Rationale for intervention activities directed at the neonate}

- To reduce stress experienced by the neonate

- Activities can assist in conserving the neonate's energy for growth

- To respond appropriately to the neonate's behaviour

- To understand the neonate's current level of organization

- To modify care according to the neonate's behaviour and ability to organize himself

- To adapt to the neonate's developing individual needs

\section{Intervention directed at the parents (Rossetti, 2001; Güldenpfennig, 2000)}

\section{Strategies for intervention}

- Determine the parents' specific circumstances

- Establish a relationship with the parents, accept their diversity and support them

- Determine the parents' knowledge and experience about preterm birth

- Train parents to develop skills to respond appropriately to the neonate, keep a journal, reflect on their own feelings and use available resources

- Guide parents during the stages of attachment

- Focus on parental and; family strengths

- Provide information aḅout developmental expectations and the need for serial developmental assessments after discharge

,

\section{Rationale for intervention activities directed at the parents}

- To reduce the stressful impact of the NICU on the neonate and family

- To facilitate adaptive parenting and overcome difficulties with attachment to the neonate

- To respect personal, social and cultural influences on the family

- To ensure independent home care after discharge from the NICU

\section{Intervention directed at the NICU staff (Rossetti, 2001)}

- To be aware of the environment in terms of noise, lighting, handling and positioning of the neonates

- To identify stress signs in the neonates and respond appropriately

- To identify neonatal developmental stages and promote self regulating behaviour in infants

- To implement developmental care effectively

- To apply new strategies and trends in developmental care, such as kangaroo mother care

- To integrate services provided by different disciplines

- To promote the social environment in the NICU 